\title{
Applications of Polysomnography and APNEA-GRAPH in Obstructive Sleep Apnea
}

\author{
Lyudmil Matev \\ Department of Dental Materials Science and Propaedeutics of Prosthetic Dental Medicine, \\ Faculty of Dental Medicine, Medical University of Varna, Bulgaria
}

\begin{abstract}
Polysomnography is a golden standard in the diagnosis of obstructive sleep apnea (OSA). During the recent several years, numerous investigations devoted to the diagnostic value of this complex method in children and adults presenting with OSA and snoring have emerged. The publications proving the effective applicability of polysomnography in dental medicine and, especially, in orthodontics, are of undoubted interest.

Apnea Graph is a modern tool for sleep analysis in ambulatory setting. By using this method, the localization of airway obstruction during snoring and OSA as the type of sleep apnea as obstructive or central can be defined. Apnea Graph is applied not only alone but also following polysomnography and in combination with other clinical and apparatus diagnostic methods. It is of particular value in screening investigations.
\end{abstract}

Keywords: obstructive sleep apnea, diagnosis, polysomnography, Apnea Graph

\section{Introduction}

Polysomnography is stated to be the gold standard in the diagnosis of sleep disordered breathing in adults $(8,14$ and others) and children $(15,22$ and others).

Polysomnography is performed overnight and is generally divided in two groups - laboratory(incenter overnight sleep study) and ambulatory(outof-center sleep testing) type(for strictly selected patients) (4). Home sleep apnea testing is a type of out-of-center sleep testing. Attended polysomnography includes at least 7 channels of recording, including electroencephalography (EEG), electrocardiography, and monitoring of sleep, airflow through the nose and mouth, pulse oximetry, respiratory effort, and leg movement. Home sleep apnea testing includes 4-7 channels of recording.

American Association of Orthodontists (AAO) tasked medical and dental experts in sleep medicine to create a document offering guidance to practicing orthodontists engaged with the management of OSA. (4)

\section{Polysomnography in childhood}

27 children, aged between 5.2 and 6.1 years (mean 6 years), polysomnography - diagnosed with OSA/ OSAS, were enrolled in a study. (11) Statistically significantly higher upper intercanine and intermolar distances are found in children with severe OSAS grade, compared to mild and moderate grades. 
Overnight polysomnography proved OSA in 11 out of 18 children with Down syndrome (mean age $11,5 \pm 2,2$ years). (6) Children with OSA displayed statistically significantly higher levels of gingival index GI $(P=0.020)$ and bleeding on probing index $\operatorname{BOP}(P=0.006)$.

Polysomnography proved OSAS in 9 out of 52 children (aged 2,5), recruited to a study, suffering from different anomalies in craniofacial and occlusal development (12). Additional otorhinolaryngological and dental examinations of those pediatric patients showed narrower intercanine width $(\mathrm{p}=$ $0,032)$, larger adenoid size with respect to the nasopharyngeal volume $(\mathrm{p}=0,020)$ and more tendency to mouth breathing $(\mathrm{p}=0,002)$ compared to nonsnoring children.

Another study involved 31 children with polysomnography-proven OSA. They were evaluated against 36 control patients with low risk for having sleep problems. Results showed significantly worsened oral health in children with OSA compared to the control group (18). Cases of dental caries are more frequent $(15,2$ versus 3,$2 ; p<0,001)$ and periodontitis (with bleeding on probing index of $87 \%$ versus $30 \%$; $<<0,001$ and more sites with abnormally deep periodontal probing depths $-2,7$ versus 0,$3 ; p<0,001)$.

131 pediatric patients of mean age $101,85 \pm 59,15$ months were evaluated, 69 of whom were males and 62 females.(3) 59 complained of snoring and 52 - of OSA. Mean obstructive hypopnea-apnea index was 5.12 \pm 11.72 . Prevalence of OSA was 33,59\%. Mean obstructive hypopnea-apnea index of patients experiencing apnea during sleep was significantly higher than the mean obstructive hypopnea-apnea index of the children who do not experience apnea $(7.52 \pm 14.25$ versus $2.61 \pm 5.84$, $\mathrm{p}=0,008)$ in OSA patients and in snoring ones $(6,93 \pm 13,53$ versus $2,32 \pm 5,43 ; p=0,011)$.

Respiratory disturbance was seen in $90 \%$ of the 30 pediatric polysomnography-diagnosed OSApatients, enrolled in a study in Iran. 16 were females and 14 - males, aged between 6 and 18 years. (19) Symptoms included snoring (in 18 of them), frequent awakening (in 17), nocturnal sweating(in 15), daytime sleepiness (in 12), sleep talking (in 10), bedtime resistance (in 9), having nightmares (in 8), waking up problems (in 6), sleep walking (in 6), difficult breathing (in 4), bedwetting (in 3) and sleep onset delay - in 2 of the patients. Severe, moderate and mild apnoea- hypopnoea indices were observed in 12, 9 and 6 of the children respectively.

OSA was proven with full-night polysomnography in $170(55 \%)$ out of 310 pediatric patients, aged between 2 and 18 years (mean age $-7,6 \pm 3,7$ years). (7) Optimal points of all indicators(age, tonsil and adenoid size, obesity and apnoeic episodes) for predicting apnoea-hypopnoea index of $>1$ and $>5$ were 10 (sensitivity, 72,9\%; specificity, $65,0 \%$ ) and 12 (sensitivity, 77,5\%; specificity, 56,9\%).

A total of 1115 children were included in another study (21) and underwent polysomnography examination. They were divided into 4 groups: group 1 - 143 with OSAS (obstructive apnea hypopnea index $\geq 1$ ); group $2-345$ with primary snoring (obstructive apnea hypopnea index $<1$ ); group 3 -529 with obstructive apnea hypopnea index $>5$ or obstructive apnea index $>1$ and group $4-98$ healthy children as a control group. After adjustments for age, gender and body mass index, children in group 1 had higher total sleep disorder scale score $(\mathrm{p}<0,01)$, as well as sub-scores for severe snoring $(\mathrm{p}<0,01)$, daytime behavior problem $(\mathrm{p}<$ $0,01)$ and sleep related nighttime abnormality $(\mathrm{p}<$ $0,05)$, compared with normal controls.

\section{Polysomnography in adulthood}

A cross-sectional study was conducted in Kingdom of Saudi Arabia in order to diagnose OSA with polysomnography. (2) A total of 51 non-obese adult patients were enrolled, 31 males and 20 females, mean age of $49,45 \pm 10,35$ years. OSA was moderate in 17 subjects, but 34 were diagnosed with a severe form of OSA. Neither the demographic characteristics, nor characteristics of dental occlusion showed statistically significant relationship with the severity of OSA.

199 patients of mean age 49,9 years, 107 females and 92 males, were enrolled in a study. (10) $62,3 \%$ were diagnosed with periodontitis, and $34,1 \%$ - with gingivitis. Statistically significant association was shown between periodontitis and mild polysomnography-proven $\operatorname{OSA}(\mathrm{p}=0,041)$. Further analysis of the results showed a statistically significant difference for patients with OSA and arterial hypertension $(\mathrm{p}<0,001)$ and those with OSA and hypertensive cardiomyopathy $(\mathrm{p}<0,001)$ compared with healthy individuals. Probably periodontitis was more likely in men with severe OSA and with any of two comorbidities.

Glossopharyngeal airway obstruction in 125 patients 
diagnosed with moderate-to-severe OSAHS was assessed using polysomnography(after nasopharyngeal tube insertion) and using spiral computed tomography. (17) Neither apnea-hypopnea index values nor lowest oxygen saturation values obtained from the first polysomnography were significantly correlated with the cross-sectional area or the inner diameter of the glossopharyngeal airway. Apneahypopnea index values after nasopharyngeal tube insertion were significantly correlated with the cross-sectional area or the inner diameter (with correlation coefficients of 0,$264 ; p=0,039$ ) and anteroposterior diameter of the glossopharyngeal airway (with correlation coefficients of 0,$185 ; \mathrm{p}=$ $0,003)$. Obvious glossopharyngeal airway stenosis was proven using both methods in 52 patients, and with CT scan only - in 41 subjects. The two methods reached the same diagnosis in 86 cases, with a consistency rate of $68.8 \%$.

\section{Apnea Graph}

Apnea Graph is a modern tool for sleep analysis in ambulatory setting. By using this method, the localization of airway obstruction during snoring and OSA as the type of sleep apnea as obstructive or central can be defined. Apnea Graph is applied not only alone but also following polysomnography and in combination with other clinical and apparatus diagnostic methods measuring nasopharyngeal air pressure. It is of particular value in screening investigations. Some of the essential features of Apnea Graph include: sleep analysis according to polysomnography; localization of airway obstruction during the natural sleeping pattern improving diagnostics and results from surgical treatment; no technical assistants to perform the examination or sleep medicine doctors to interpret the results are required. Apnea Graph is done in ambulatory setting and stands to be economically more practical than the conventional polysomnography.

16 patients, 12 of whom males and 4 females, all suffering from OSA (OSAS) underwent diagnostic procedures with Apnea Graph and dental examinations including cephalometry to identify the localization of the obstruction.(5) A firm correlation was established between otolaryngologic value of AHI and the cephalometric values of the ANB angle, with the distance between the hyoid bone and the mandibular plane. Caudal position of the hyoid bone is a sign for severe OSAS.

The diagnostic value of Apnea Graph regarding the localization of the obstruction and determining the stage of OSAS is compared to the value of laboratory and ambulatory polysomnography in 30 males enrolled in a clinical trial. (9) Severity stages of OSAS determined by apnea-hypopnea index using both methods are different in $44 \%$ of the cases. Such dominant collapse levels are detected in $64 \%$ of the patients.

37 patients with OSA were investigated with pulse oximetry and Apnea Graph.(1) According to the results using pulse oximetry only, 31 are diagnosed with snoring, whereas Apnea Graph showed 11 patients with a moderate degree and one with a severe degree of OSA.

50 patients with OSAHS, previously underwent polysomnography, in whom a number of indices of sleep medicine were assessed persperctively using a 2-h day - or nighttime Apnea Graph. The aim was to identify the level of obstruction before the surgical intervention. (23) All indices except the lowest oxygen saturation index found no significant differences between day- and nighttime Apnea Graph. Most moderate and severe patients had predominant sites of obstructions in the upper level.

115 patients suffering from OSAS and hypopnea, previously underwent polysomnography, were synchronously examined by Apnea Graph and micro movement sensitive mattress sleep monitoring system in Beijing, China. (20) Mild, moderate and severe OSAS grading was found with Apnea Graph in 6,16 and 93 patients, and with micro movement sensitive mattress sleep monitoring system $-8,26$ and 81 respectively. Hypoxemia severity grading - mild, moderate and severe - found with Apnea Graph in 14, 70 and 27 of the patients, and 6, 72 and 37 respectively, investigated with micro movement sensitive mattress sleep monitoring system. Parameters of apnea-hypopnea index are 51,5 $\pm 21,2$ times/h and $48,1 \pm 24,2$ times/h $(r=0,947)$, the lowest oxygen saturation index $-0,736 \pm 0,110$ and $0,710 \pm 0,108(\mathrm{r}=0,935)$, the correlation coefficient of apnea index and obstructive apnea and hypopnea index $-r=0,856$ и $r=0,841(p<0,01)$, positively correlated data obtained through the methods.

30 patients, 28 males and 2 females, with median age (ranging from 35 to 59) of 41,5, were diagnosed with severe OSAHS by overnight polysomnography. The mean body mass index was $28,8 \pm 4,1$ $\mathrm{kg} / \mathrm{m}^{2}$. Investigations with Apnea graph and cephalometry were conducted (24). Mean apnea-hypopnea index was $58,6 \pm 16,4$ times/h, lowest oxygen 
saturation was averaged to $0,69 \pm 0,09$. All patients had narrow PAS with the mean of $7,6 \pm 2,1 \mathrm{~mm}$ (between $4,4 \mathrm{~mm}$ and $10,8 \mathrm{~mm}$ ). Only in 2 of the cases obstruction of more than $50 \%$ of the airway at lower level was observed. Eight of the patients had 30 to $40 \%$ obstruction, six had less than $6 \mathrm{~mm}$ narrow PAS. Therefore patients with a higher percentage of lower obstruction had the tendency to have a corresponding narrower PAS.

A prospective study was performed, which included 14 patients suffering from OSAS. A good correlation between Apnea Graph and polysomnography measurements for apnea-hypopnea index, pulse, $\mathrm{SaO} 2$, body position and central apnea was established. (13) The specified study showed differences between the two methods for apnea index $(\mathrm{p}=0,002)$, hypopnea index $(\mathrm{p}=0,013)$, mixed apnea $(p=0,003)$ and obstructive apnea $(p=0,013)$. Apnea Graph proved a pure upper airway obstruction in 2 cases, while six patients had a predominance of lower obstruction.

A prospective study of 49 patients with snoring and/ or OSA was done with the aim to evaluate results from implementing sleep nasendoscopy, polysomnography and Apnea Graph (16). No significant differences $(p>0,15)$ between polysomnography and Apnea Graph regarding apnoea-hypopnoea index, total number of apnoeic events, average oxygen saturation and maximal desaturation.

Apnea Graph is of particular value in screening investigations among risk groups of patients for OSA.

\section{References}

1. Вълчева 3, Арнаутска X, Милков М. Устното дишане при деца и връзката му с обструктивната сънна апнея. Международен бюлетин оториноларинголия 2018; 14(1): 32.

2. Милков М. Диагностика на нарушенията на съня. - Международен бюлетин оториноларингология, Варна, 5, 2009, 4, 31-35

3. Милков М. Езофагиална манометрия - метод за топична диагностика на хъркането. Международен бюлетин оториноларингология, Варна, 6, 2010, 1, 48-52.

4. Achar P, Kumar BN. Can apnea graphy change our approach in management of snoring and sleep apnoea? Indian J Otolaryngol Head Neck Surg. 2014; 66(Suppl 1): 110-114.

5. Alqahtani ND, Algowaifly MI, Almehizia FA, Alraddadi ZA, Al-Sehaibany FS, Almosa NA, et al. The characteristics of dental occlusion in patients with moderate to severe obstructive sleep apnea in Saudi Arabia. Saudi Med J. 2018; 39(9): 928-934.

6. Baev P, Matev L, Tsonchev Ts, Milkov M. Lateral cephalometric radiographic values in diagnosing the obstructive sleep apnea. Sex differences. In: Second National Symposium on Obstructive Sleep Apnea and Snoring with International Participation. Varna, 26-27.VIII.2016. Междунар бюл оториноларингол. 2016; 12(1): 48.

7. Barış HE, Gökdemir Y, Eralp EE, İkizoğlu NB, Karakoç F, Karadağ B, et al. Clinical and polysomnographic features of children evaluated with polysomnography in pediatric sleep laboratory. Turk Pediatri Ars. 2017; 52(1): 23-29.

8. Behrents RG, Shelgikar AV, Conley RS, Flores-Mir C, Hans M, Levine M, et al. Obstructive sleep apnea and orthodontics: An American Association of Orthodontists White Paper. Am J Orthod Dentofacial Orthop. 2019; 156(1): 13-28.

9. Di Venere D, Corsalini M, Nardi GM, Laforgia A, Grassi FR, Rapone B, et al. Obstructive site localization in patients with Obstructive Sleep Apnea Syndrome: a comparison between otolaryngologic data and cephalometric values. Oral Implantol (Rome). 2017; 10(3): 295-310.

10. Durhan MA, Agrali OB, Kiyan E, Ikizoglu NB, Ersu R, Tanboga I. Does obstructive sleep apnea affect oral and periodontal health in children with Down syndrome? A preliminary study. Niger J Clin Pract. 2019; 22(9): 1175-1179.

11. Kang KT, Weng WC, Lee CH, Hsiao TY, Lee PL, Hsu WC. Clinical risk assessment model for pediatric obstructive sleep apnea. Laryngoscope. 2016; 126(10): 2403-2409.

12. Kang S, Lee Y, Lim YH, Park HK, Cho SH, Cho SH. Validation of noncontact cardiorespiratory monitoring using impulse-radio ultrawideband radar against nocturnal polysomnography. Sleep Breath. 2019 Aug 10. doi: 10.1007/s11325-019-01908-1.

13. Karaloğlu F, Kemaloğlu YK, Yilmaz M, Ulukavak Çiftçi T, Çiftçi B, Bakkal FK. Comparison of full-night and ambulatory polysomnography with ApneaGraph in the subjects with obstructive sleep apnea syndrome. Eur Arch Otorhinolaryngol. 2017; 274(1): 189-195.

14. Latorre C, Escobar F, Velosa J, Rubiano D, Hidalgo-Martinez P, Otero L. Association between obstructive sleep apnea and comorbidities with periodontal disease in adults. J Indian Soc Periodontol. 2018; 22(3): 215-220.

15. Madjova Ch, Milkov M, Panov Vl - Sleep apnea in children with obesity - First National Symposium on Obstructive Sleep Apnea and Snoring with International Participation. Varna, 16-18.10.2014

16. Matev L, Milkov M, Tonchev Ts, Pomakova A, Nedev P, Iliev G, Dikova Tz, Madjova H. Night manometry of the upper airway in snoring and obstructive sleep apnea. Oto Rhino Laryngology International bulletin, 2014;(2), 12-15, ISSN 1312-6997

17. Marino A, Nota A, Caruso S, Gatto R, Malagola C, Tecco S. Obstructive sleep apnea severity and dental arches dimensions in children with late primary dentition: An observational study. Cranio. 2019 Jun 25:1-6. doi: 10.1080/08869634.2019.1635296.

18. Markkanen S, Niemi P, Rautiainen M, Saarenpää-Heikkilä O, Himanen SL, Satomaa AL, et al. Craniofacial and occlusal development in 2.5year-old children with obstructive sleep apnoea syndrome. Eur J Orthod. 2019; 41(3): 316-321.

19. Morales Divo C, Selivanova O, Mewes T, Gosepath J, Lippold R, Mann WJ. Polysomnography and ApneaGraph in patients with sleep-related breathing disorders. ORL J Otorhinolaryngol Relat Spec. 2009; 71(1): 27-31.

20. Park DY, Kim T, Lee JJ, Ha JH, Kim HJ. Validity analysis of respiratory events of polysomnography using a plethysmography chest and abdominal belt. Sleep Breath. 2019 Oct 30. doi: 10.1007/s11325-019-01940-1.

21. Raynov A, Milkov M - Application of Medical devices in the treatment of patients with Obstructive Sleep Apnea - First National Symposium on Obstructive Sleep Apnea and Snoring with International Participation. Varna, 16-18.10.2014 
22. Savini S, Ciorba A, Bianchini C, Stomeo F, Corazzi V, Vicini C, Pelucchi S. Assessment of obstructive sleep apnoea (OSA) in children: an update. Acta Otorhinolaryngol Ital. 2019; 39(5): 289-297.

23. Singh A, Al-Reefy H, Hewitt R, Kotecha B. Evaluation of ApneaGraph in the diagnosis of sleep-related breathing disorders. Eur Arch Otorhinolaryngol. 2008; 265(12): 1489-1494.

24. Song M, Bao J, Wang X, Li S. Diagnosis of glossopharyngeal obstruction using nasopharyngeal tube versus CT scan in obstructive sleep apnea-hypopnea syndrome. Eur Arch Otorhinolaryngol. 2015; 272(5): 1175-1180.

25. Tamasas B, Nelson T, Chen M. Oral health and oral health-related quality of life in children with obstructive sleep apnea. J Clin Sleep Med. 2019; 15(3): 445-452.

26. Tavasoli A, Jalilolghadr S, Lotfi S. Sleep symptoms and polysomnographic patterns of obstructive sleep apnea in obese children. Iran J Child Neurol. 2016; 10(1): 14-20.

27. Tian X, Li W, Huo H, Yu R, Wang J. The clinical significance of synchronous monitoring with micro-movement sensitive mattress sleep monitoring system and Apnea Graph. Lin Chung Er Bi Yan Hou Tou Jing Wai Ke Za Zhi. 2013; 27(18): 1016-1020.

28. Xu ZF, Wu YX, Feng GS, Zhang YM, Ni X. Evaluation of polysomnographic diagnostic criteria for obstructive sleep apnea syndrome in children. Zhonghua Er Bi Yan Hou Tou Jing Wai Ke Za Zhi. 2016; 51(11): 806-811.

29. Yan XH, Zhao Y, Wang J, Shen T, Yang W, Qiao Y, et al. Associations among sleep symptoms, physical examination, and polysomnographic findings in children with obstructive sleep apnea. Eur Arch Otorhinolaryngol. 2019 Nov 8. doi: 10.1007/s00405-019-05719-8.

30. Yu R, Li W, Huo H, Shen P, Tian X. Short daytime ApneaGraph for initial case selection of obstructive sleep apnea-hypopnea syndrome before surgery. Eur Arch Otorhinolaryngol. 2011; 268(11): 1663-1669.

31. Vasileva Sv, Benchev R, Stoyanov S, Mikov M - Metabolic, endocrine and systemic disorders associated with the syndrome of obstructive sleep apnea(OSA) - First National Symposium on Obstructive Sleep Apnea and Snoring with International Participation. Varna, 1618.10.2014

32. Zhou CY, Dai ZY, Li YF, Wen YL, Zong L, Zhang H. Correlation study between obstructive level diagnosed by Apnea Graph and cephalometric posterior airway space in obstructive sleep apnea hypopnea syndrome. Zhonghua Er Bi Yan Hou Tou Jing Wai Ke Za Zhi. 2011; 46(8):617621

\section{Correspondence address: \\ Assist. Prof. Lyudmil Matev, MD \\ ES Propaedeutics of Prosthetic Dental Medicine}

Department of Dental Material Science and Propaedeutics of Prosthetic Dental Medicine

Faculty of Dental medicineq Medical University "Prof. Dr. Paraskev Stoyanov" - Varna

Varna 9002

84 Tsar Osvoboditel blvd.

E-mail:lyudmil.matev@mu-varna.bg 